\title{
edoc
}

Institutional Repository of the University of Basel

University Library

Schoenbeinstrasse 18-20

CH-4056 Basel, Switzerland

http://edoc.unibas.ch/

Year: 2013

\section{Associations between mental disorders and the common cold in adults: a population-based cross-sectional study}

\author{
Yuki Adam, Gunther Meinlschmidt, Roselind Lieb
}

Posted at edoc, University of Basel

Official URL: http://edoc.unibas.ch/dok/A6168343

Originally published as:

Adam, Y., Meinlschmidt, G. \& Lieb, R. (2013). Associations between mental disorders and the common cold in adults: a population-based cross-sectional study. Journal of Psychosomatic Research, 74, 1, 69-73.

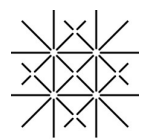


Associations between mental disorders and the common cold in adults: a population-based cross-sectional study

Yuki Adam, MSc ${ }^{\text {a }}$, Gunther Meinlschmidt, PhD ${ }^{\text {a, b, c }}$, Roselind Lieb, PhD ${ }^{\text {a, b, * }}$

${ }^{a}$ National Centre of Competence in Research "Swiss Etiological Study of Adjustment Health and Mental Health", Department of Psychology, University of Basel, Basel, Switzerland

b Division of Clinical Psychology and Epidemiology, Department of Psychology, University of Basel, Basel, Switzerland

c Research Department of Psychobiology, Psychosomatics, and Psychotherapy, Clinic of Psychosomatic Medicine and Psychotherapy, LWL University Hospital, Ruhr-University Bochum, Bochum, Germany.

* Corresponding author: Division of Clinical Psychology and Epidemiology, Department of Psychology, University of Basel, Missionsstrasse 60/62, 4055 Basel, Switzerland. Phone: +41 61/267 02 78; Fax: +41/61 2670274.

E-Mail: roselind.lieb@unibas.ch (R. Lieb).

A short running head: Mental disorders and the common cold

Words count abstract: 244

Total number of words (Introduction through Discussion): 3054

Number of tables: 2 


\section{ABSTRACT}

Objective: To investigate the association between specific mental disorders and the common cold.

Methods: Negative binomial regression analyses were applied to examine crosssectional associations of a broad range of mental disorders according to the Diagnostic and Statistical Manual of Mental Disorders, Fourth Edition (DSM-IV) employing the standardized diagnostic Munich Composite International Interview, with the self-reported number of occurrences of the common cold during the past 12 months in a representative population sample of 4022 German adults aged 18-65 years.

Results: After adjustment for covariates including age, gender, and marital and socioeconomic status, having any 12-month DSM-IV mental disorder (incidence rate ratio $[\mathrm{IRR}]=1.44,95 \%$ confidence interval $[\mathrm{Cl}]=1.29-1.60)$, any substance abuse or dependence $(\mathrm{IRR}=1.32,95 \% \mathrm{Cl}=1.14-1.52)$, possible psychotic disorder $(\mathrm{IRR}=$ $1.43,95 \% \mathrm{Cl}=1.09-1.87)$, any mood disorder $(\mathrm{IRR}=1.35,95 \% \mathrm{Cl}=1.16-1.56)$, any anxiety disorder (IRR $=1.40,95 \% \mathrm{Cl}=1.23-1.59)$, or any somatoform disorder $(\mathrm{IRR}=1.38,95 \% \mathrm{Cl}=1.18-1.62)$ was shown to be positively associated with the number of occurrences of a cold during the past 12 months.

Conclusion: The presence of a DSM-IV mental disorder was associated with a $44 \%$ higher risk of having experienced a cold in the past 12 months. Further studies are needed to explore potential common risk factors for incidence of mental disorders and the common cold, since the pathway connecting them has not been fully determined.

Keywords: common cold, cross-sectional, mental disorder, population-based sample 


\section{Introduction}

Several studies have revealed that poor mental health conditions including perceived stress [1-3] and negative mood [2, 4, 5] are risk factors for developing a common cold. However, prior data relate to symptom scales rather than diagnoses of mental disorders. As yet, it is unclear whether the presence of a mental disorder has an impact on susceptibility to the common cold.

The link of mental disorders with the common cold is an important public health matter. Both mental disorders and the common cold are prevalent in general populations and are associated with a high economic burden in terms of work absenteeism and health care costs [6-8]. Recent estimates indicate that every year over $38.2 \%$ of the total European population of 30 countries, or 164.8 million people, suffers from at least one mental disorder [6]. In addition, the common cold is one of the most frequent infectious illnesses [9]. It has been reported that adults experience on average two to five colds per person each year [10]. Symptoms of the common cold, including nasal stuffiness and discharge, sore throat, chills, cough, headache, and malaise, are so common that tests are of no use in diagnosing the common cold [9]. More than 200 types of viruses have been detected as being responsible for colds, yet up to $30 \%$ of all colds still remain without a proven viral cause [9].

Available knowledge of the impact of mental health on the vulnerability to upper respiratory infections largely relies on experimental studies, in which subjects fulfilling certain health conditions were exposed to viruses [3]. According to the findings from the World Mental Health Surveys [11], growing numbers of people frequently experience co-occurrence of physical and mental disorders. Examining the relationship of mental disorders and occurrences of the common cold in a general population is therefore an urgent issue. 
The present study investigates whether, and to what extent, a broad range of DSM-IV (Diagnostic and Statistical Manual of Mental Disorders, Fourth Edition) [12] mental disorders (i.e., substance, mood, anxiety, and somatoform disorders), diagnosed with the standardized diagnostic Munich Composite International Diagnostic Interview (M-CIDI) [13], are associated with subjects' self-reported number of occurrences of the common cold during the past 12 months in a representative population sample aged 18-65 years.

\section{Methods}

Design

We used data from the German National Health Interview and Examination Survey (GHS) [14]. The GHS was conducted to supply representative nationwide epidemiologic data on major mental and physical disorders, impairments, and healthcare utilization. The GHS was based on a stratified, cross-sectional sample randomly drawn from the population registries of individuals living in Germany in 1997. Data were collected between November 1997 and April 1999 [14]. The GHS was commissioned by the German Ministry of Research, Education, and Science and the Robert Koch Institute and approved by the relevant institutional review board and ethics committee. All subjects provided written informed consent. A detailed description of the GHS methodology and sampling is available elsewhere [14]. Data of the present study are based on the sample of the GHS Mental Health Supplement (GHS-MHS) that was conducted in individuals aged 18 to 65 years ( $n=4181$; conditional response rate $87.6 \%$ from $N=4775$ eligible subjects) $[14,15]$. There were no differences between the respondents and nonrespondents in terms of gender, age, and self-reported health status [14]. 


\section{Measures}

\section{Mental disorders}

The computer-assisted version of the Munich Composite International Diagnostic Interview (DIA-X/M-CIDI) [13], administered by clinically trained psychologists and physicians, was employed in assessments of mental disorder diagnoses in the GHS-MHS. The fully structured DIA-X/M-CIDI is a modified version of the World Health Organization CIDI (version 1.2), supplemented by questions to cover DSM-IV and International Classification of Diseases, version 10 (ICD-10) criteria. Mental disorder diagnoses reported in the study were obtained using the DIA-X/M-CIDI diagnostic algorithms for 12-month DSM-IV criteria [13]. Details of psychometric properties and feasibility of the DIA-X/M-CIDI are available elsewhere $[16,17]$. In summary, the test-retest reliability of the full DIA-X/M-CIDI was good, with kappa values ranging between 0.56 and 0.81 [16]. The validity of the full DIA-X/MCIDI diagnoses compared to diagnoses from independent treating physicians in a sample of 68 randomly chosen patients ranges from moderate to excellent [16]. The sensitivity of the DIA-X/M-CIDI mental disorder diagnoses ranges from $87.5 \%$ to $100 \%$, while their specificity ranges from $71.2 \%$ to $100 \%$ [17].

The present study included the following 12-month DSM-IV mental disorder diagnostic categories: substance use disorders (alcohol abuse or dependence, nicotine dependence, illicit substance abuse or dependence), possible psychotic disorder, mood disorders (major depression, dysthymia, bipolar disorders), anxiety disorders (panic disorder, agoraphobia, social phobia, specific phobia [animal, natural environment, blood-injection-injury, situational type], generalized anxiety disorder, obsessive-compulsive disorder), and somatoform syndromes and disorders (somatic symptom index 4/6, pain disorder). 


\section{Number of occurrences of a cold}

All subjects were asked to report the number of occurrences of a cold during the previous 12 months with the question, "In the previous 12 months, did you have once or several times an influenza or a common cold, so that you could not go to work or carry out your daily activities?" (no, once, several times). If subjects reported having had an influenza or a common cold several times during the past 12 months, they were subsequently asked to report how often an influenza or a common cold had occurred in that period.

\section{Sociodemographic information}

We considered gender, age, and marital and socioeconomic status as covariates because these have been linked with both mental disorders and common cold occurrences in previous studies $[1,15,18]$. Age was treated as a continuous measure. Marital status was dichotomized (in relationship, not in relationship). Socioeconomic status was dichotomized according to the Winkler Index [19] that uses information about educational level, monthly net household income, and occupational position as indicators (middle/high, low).

\section{Statistical analyses}

The dependent variable was the number of occurrences of a cold during the previous 12 months. A count variable has certain properties: (a) it takes on whole numbers only, (b) it can never be negative, and (c) it tends to be positively skewed [20]. Since a count variable often does not meet the assumptions of normality and homoscedasticity which is required for ordinary least squares regression [21] a Poisson model is often preferred. The Poisson model, however, assumes that the 
variance of the counts is equal to the mean [20]. In the case of over-dispersion, i.e., when the variance of the dependent variable is larger than the mean, the negative binomial model, which is an extension to the Poisson model as it captures the extent of overdispersion, is generally better suited [21]. As the number of occurrences of a cold showed considerable overdispersion, we used negative binomial regression model to analyse the associations between the DSM-IV mental disorders (predictors) and the self-reported number of occurrences of a cold (outcome) during the previous 12 months. Model coefficients are reported in terms of incidence rate ratios (IRRs) with 95\% confidence intervals (Cls), because parameter estimates based on the analysis of counts are generally expressed in terms of IRRs [21]. IRR here denotes by which factor the estimated number of occurrences of a cold is increased if having a specific DSM-IV mental disorder [21]. In addition, we checked for multicollinearity among all variables considered in the study. An increased degree of multicollinearity (i.e., an inflation factor greater than 10) may cause a risk for the unstable estimation of the regression coefficients [22]. Variances of inflation factor values of the present data were unproblematic, ranging from 1.01 to 1.26 .

Covariates in the analysis included gender, age, and marital and socioeconomic status. The appropriate statistical weight was applied in all analyses to ensure that the data were representative of the population [14]. A $p$ value $<.05$ was considered statistically significant. Analyses were performed using STATA 10.0 [23].

Study subjects

A total of 4181 GHS-MHS subjects were eligible for this study objective. Of these, 126 subjects were excluded from the study due to missing data; in 90 subjects 
the number of occurrences of a cold was not reported and 36 subjects did not report their marital and/or socioeconomic status. Among the 4055 subjects with complete data that were relevant to the study, we detected multivariate outliers. We defined an outlier as an observation whose dependent-variable value was unusual given its values on the predictor variables, which may have been caused by a sample peculiarity or a data entry error [24]. Since the outliers may have had an overall impact on the estimations, we examined Studentized residuals and their distributions with plots. We detected 33 subjects with absolute Studentized residuals greater than 3 as outliers and excluded them from the analyses. The present analyses were done on a total of 4022 subjects aged 18 to 65 years (96.9\% of the GHS-MHS subjects).

\section{Results}

Among the 4022 subjects included in the study, 2021 were male (50.3\%). The mean age of the study subjects was 41.8 years $(S D=13.1)$. The majority were in a relationship ( $n=2583 ; 64.2 \%$ ), and 3265 subjects $(81.2 \%$ ) of the study sample had middle or high socioeconomic status. A proportion of $1.3 \%(n=52)$ of the study sample received treatment in a psychiatric hospital at any point in their lives. Table 1 provides the sociodemographic characteristics of the sample. The study subjects reported a total of 1563 occurrences of a cold (mean $=0.55 ; \mathrm{SD}=0.83$; range $0-5$ episodes) during the past 12 months.

\section{- Table 1 about here -}

Overall, subjects with any one of the explored $D S M-I V$ mental disorders had a higher IRR of colds than the reference subjects (i.e., subjects without any of the explored DSM-IV mental disorder) during the past 12 months (see Table 2). After adjustments for sociodemographic covariates, a $44 \%$ higher risk of having 
experienced a cold was found in subjects with any DSM-IV mental disorder compared to subjects with no DSM-IV mental disorders (IRR $=1.44,95 \% \mathrm{Cl}=1.29$ 1.60). Likewise, a risk of having experienced a cold was $43 \%$ higher in subjects with a possible psychotic disorder compared to subjects without a possible psychotic disorder $(\mathrm{IRR}=1.43,95 \% \mathrm{Cl}=1.09-1.87), 40 \%$ higher in subjects with any anxiety disorder compared to subjects without any anxiety disorder $(\mathrm{IRR}=1.40,95 \% \mathrm{CI}=$ 1.23-1.59), 38\% higher in subjects with any somatoform disorder compared to subjects without any somatoform disorder (IRR $=1.38,95 \% \mathrm{Cl}=1.18-1.62), 35 \%$ higher in subjects with any mood disorder compared to subjects without any mood disorder $(\mathrm{IRR}=1.35,95 \% \mathrm{Cl}=1.16-1.56)$, and $32 \%$ higher in subjects with any substance abuse or dependence disorder compared to subjects without any substance abuse or dependence disorder (IRR $=1.32,95 \% \mathrm{Cl}=1.14-1.52)$ in the adjusted models.

With regard to relationships between each 12-month DSM-IV mental disorder and colds, presence of nicotine dependence (IRR $=1.28,95 \% \mathrm{Cl}=1.10-1.50)$, illicit substance abuse or dependence (IRR $=2.39,95 \% \mathrm{Cl}=1.73-3.30$ ), major depression $(\mathrm{IRR}=1.39,95 \% \mathrm{Cl}=1.17-1.64)$, dysthymia $(\mathrm{IRR}=1.34,95 \% \mathrm{Cl}=$ 1.07-1.68), panic (IRR $=1.57,95 \% \mathrm{Cl}=1.19-2.08)$, agoraphobia $(\mathrm{IRR}=1.47,95 \%$ $\mathrm{Cl}=1.06-2.04)$, specific phobia $(\mathrm{IRR}=1.52,95 \% \mathrm{Cl}=1.29-1.78)$, somatic symptom index $4 / 6(\mathrm{IRR}=1.39,95 \% \mathrm{Cl}=1.09-1.76)$, and pain $(\mathrm{IRR}=1.26,95 \% \mathrm{Cl}=1.05-$ 1.51) remained related to the number of occurrences of a cold, even after adjusting for sociodemographic covariates. There were no relationships between alcohol abuse or dependence or bipolar, social phobia, generalized anxiety, or obsessivecompulsive disorder and the number of occurrences of a cold.

- Table 2 about here - 


\section{Discussion}

This study investigated the relationship between a broad range of DSM-IV mental disorders and the self-reported number of occurrences of the common cold during the past 12 months. Unlike previous research estimating associations of colds and mental health by using self-reports of mental health conditions related to symptom scales [1-5], this study used DSM-IV mental disorder diagnoses that were assessed with a well-established standardized diagnostic interview, administered by trained clinical interviewers. It is also noteworthy that our findings are based on data from a representative population sample, whereas earlier findings about the effect of mental health on the vulnerability to colds mostly relied on results from experimental studies that used subjects fulfilling certain health criteria. Consequently, this study was not limited by sampling selection bias and extends available knowledge on the association between mental health conditions and the common cold.

The present study supports findings from literature reporting the impact of mental health conditions on the occurrence of the common cold [1-5]. We found that subjects with a $D S M-I V$ mental disorder had a $44 \%$ higher risk of having experienced a cold compared to those with no DSM-IV mental disorders during the past 12 months after adjustment for sociodemographic variables. Additionally, elevated risk for the occurrences of a cold was found in the presence of possible psychotic (43\%), any anxiety (40\%), any somatoform (38\%), any mood (35\%), and any substance use disorder (32\%) in the adjusted models. Mental disorders with the highest-ranking risk of the occurrence of a cold were illicit substance disorder (139\%), panic disorder (57\%), and specific phobia (52\%). Until now, presence of depression has received more attention than other mental disorders when examining the relationship between 
mental health and infectious outcome $[25,26]$. Therefore, future studies are needed to explore the role of a broad range of mental disorders in vulnerability to colds.

The question arises as to whether our findings indicate that (a) individuals with mental disorder are more susceptible to colds, (b) they have greater sensitivity to perceive symptoms of colds, (c) they have a tendency to over-report the occurrence of a common cold, (d) a common cold increases the risk for developing certain mental disorders, or (e) a common cold and certain mental disorders may share yet unknown common risk factors. There are several possible explanations for these five models.

First, psychological stress has been suggested to contribute to mental disorders [27]. Mental disorders may increase psychological stress and negative emotions in individuals as a result of considerable impairment to fulfilling daily functioning [28]. Therefore, presence of mental disorders may lead to a maladaptive immune system in individuals [29]. Additionally, emotional states have been found to affect individuals' engagement in health-promoting behaviors [30, 31]. Consequently, it is possible that individuals with a mental disorder are more likely to practice behaviors that are related to susceptibility to the common cold (i.e., less physical activity, poor sleeping habits, smoking). Second, it has been reported that negative emotions affect the cognitive perception of physical symptoms in individuals [32, 33]. It is possible that individuals with a mental disorder may interpret vague physical impressions more frequently as symptoms of colds compared to individuals without a mental disorder. Third, Cohen and colleagues [34] have noted that negative emotional style was associated with an over-reporting of unverified symptoms of colds, whereas positive emotional style was associated with an under-reporting of verified symptoms of colds. Although a high validity of the self-assessments of the 
cold by subjects themselves compared with the assessments by physicians has been reported [35], possible inaccuracy in self-report assessment of colds without clinical and biological observation should be noted to interpret our findings. Fourth, Dantzer and colleagues [36] have suggested that pro-inflammatory cytokines, which can be induced by infectious agents, initiate "sickness behavior." Sickness behavior, including weakness, malaise, fatigue, restlessness, sleep changes, fever, and reduced appetite, starts as a disease-coping strategy that promotes an effective immune response in the host to clear infectious agents [36]. However, prolonged or exacerbated sickness behavior may foster the development of symptoms of depression in vulnerable individuals [36]. Since our understanding of the possible contribution of infectious processes to the occurrence of mental disorders is still in its infancy [36], further research is needed to scrutinize this relationship. Fifth, although high prevalence of anxiety and depression in individuals with chronic breathing disorders has been reported [37-39], the pathophysiological mechanisms underlying these relationships remain unclear [40]. As several researchers have suggested [4144], there is a need to routinely employ screening tools for anxiety and depression in nonpsychiatric medical settings. Anxiety and depression are associated with an increased risk of relapse rates in patients with chronic breathing disorders after treatment [38, 45]. Additionally, there is a report documenting that pulmonary rehabilitation has provided improvement in both physical performance and levels of anxiety and depression in patients with chronic obstructive pulmonary disease [46]. Closer interdisciplinary cooperation between physical and mental health care settings may help to determine possible common risk factors for mental disorders and colds.

There are limitations to this study that should be mentioned. (1) The observational nature of this study does not allow for examination of the mechanisms 
by which mental disorders and colds are linked. (2) The cross-sectional nature of this study precludes determination of causality. A prospective study is needed to determine whether onset of mental disorders precedes the occurrences of a cold or whether incidence of a cold is a risk factor for the development of mental disorders, or whether mental disorders and colds have a transactional relationship. (3) Although we controlled for potential effects of sociodemographic variables, other unknown confounders that may influence the observed associations may remain unaccounted for. (4) We evaluated the association between one predictor and one outcome. However these two factors are ultimately linked, the development of a mental disorder as well as the development of colds surely is influenced by multiple risk factors and cannot be completely explained by a simple one-factor model. Therefore, the associations we found in our study may not be translated into one-factor disease models. Rather, they argue for the importance of these specific factors within complex models.

Within the context of these limitations, the current study extends prior findings by providing evidence that mental disorders are associated with the occurrence of colds. Our findings are compatible with recent findings from the World Mental Health Surveys [11] that revealed a growing number of individuals worldwide reporting frequent co-occurrence of mental and physical disorders. Further studies are needed to explore potential common risk factors for incidence of mental disorders and the common cold, since the pathway connecting mental health and susceptibility to the common cold has not been fully determined.

\section{Conflict of interest}

The authors declare no conflicts of interest. 


\section{Acknowledgements}

The GHS was supported by grant 01EH970/8 (German Federal Ministry of Research, Education, and Science). Mental disorders were assessed with the mental health supplement of the GHS (Max Planck Institute of Psychiatry, Munich, Germany; principal investigator: H.-U. Wittchen) and physical conditions and social variables with the core survey of the GHS (Robert Koch Institute, Berlin, Germany; principal investigators: B.-M. Kurth, W. Thefeld). A public use file from the data set of the core survey can be ordered from the Robert Koch Institute (Public Use File BGS98, Bundes-Gesundheitssurvey 1998, Robert Koch Institute, Berlin, 2000) and from the mental health supplement from F. Jacobi. Preparation of the paper (Yuki Adam) as part of the National Centre of Competence in Research's "Swiss Etiological Study of Adjustment Health and Mental Health" was supported by grant 51A 240-104890 from the Swiss National Science Foundation. 


\section{References}

[1] Cobb JM, Steptoe A. Psychosocial stress and susceptibility to upper respiratory tract illness in an adult population sample. Psychosom Med 1996;58:404-12.

[2] Cohen S, Tyrrell DA, Smith AP. Negative life events, perceived stress, negative affect, and susceptibility to the common cold. J Pers Soc Psychol 1993;64:131-40.

[3] Takkouche B, Regueira C, Gestal-Otero JJ. A cohort study of stress and the common cold. Epidemiology 2001;12:345-9.

[4] Kim HC, Park SG, Leem JH, Jung DY, Hwang SH. Depressive symptoms as a risk factor for the common cold among employees: A 4-month follow-up study. J Psychosom Res 2011;71:194-6.

[5] Shinkawa M, Yanai M, Yamaya M, Matsui T, Sasaki H. Depressive state and common cold. Lancet 2000;356:942.

[6] Wittchen HU, Jacobi F, Rehm J, Gustavsson A, Svensson M, Jonsson B, et al. The size and burden of mental disorders and other disorders of the brain in Europe 2010. Eur Neuropsychopharmacol 2011;21:655-79.

[7] Chevalier A, Luce D, Blanc C, Goldberg M. Sickness absence at the French National Electric and Gas Company. Br J Ind Med 1987;44:101-10.

[8] Goetzel RZ, Long SR, Ozminkowski RJ, Hawkins K, Wang S, Lynch W. Health, absence, disability, and presenteeism cost estimates of certain physical and mental health conditions affecting U.S. employers. J Occup Environ Med 2004;46:398-412.

[9] Heikkinen T, Jarvinen A. The common cold. Lancet 2003;361:51-9. 
[10] Monto AS. Epidemiology of viral respiratory infections. Am J Med 2002;112 Suppl 6A:4S-12S.

[11] von Korff MR. Global perspectives on mental-physical comorbidity. In: von Korff MR, Scott KM, Gureje O, editors. Global perspectives on mentalphysical comorbidity in the World Health Organization World Mental Health Surveys. Cambridge: Cambridge University Press; 2009, p. 1-14.

[12] American Psychiatric Association. Diagnostic and Statistical Manual of Mental Disorders, Fourth Edition. Washington, DC: American Psychiatric Association; 1994.

[13] Wittchen HU, Pfister H. DIA-X-Interviews: Manual für Screening-Verfahren und Interview; Interviewheft Längsschnittuntersuchung (DIA-X-Lifetime); Ergänzungsheft (DIA-X-Lifetime); Interviewheft Querschnittuntersuchung (DIA-X-12Monate); Ergänzungsheft (DIA-X-12Monate); PC-Programm zur Durchführung des Interviews (Längs- und Querschnittuntersuchung); Auswertungsprogramm. Frankfurt: Swets \& Zeitlinger; 1997.

[14] Jacobi F, Wittchen HU, Holting C, Sommer S, Lieb R, Hofler M, et al. Estimating the prevalence of mental and somatic disorders in the community: aims and methods of the German National Health Interview and Examination Survey. Int J Methods Psychiatr Res 2002;11:1-18.

[15] Jacobi F, Wittchen HU, Holting C, Hofler M, Pfister H, Muller N, et al. Prevalence, co-morbidity and correlates of mental disorders in the general population: results from the German Health Interview and Examination Survey (GHS). Psychol Med 2004;34:597-611.

[16] Wittchen HU, Lachner G, Wunderlich U, Pfister H. Test-retest reliability of the computerized DSM-IV version of the Munich-Composite International 
Diagnostic Interview (M-CIDI). Soc Psychiatry Psychiatr Epidemiol 1998;33:568-78.

[17] Reed V, Gander F, Pfister H, Steiger A, Sonntag H, Trenkwalder C, et al. To what degree does the Composite International Diagnostic Interview (CIDI) correctly identify DSM-IV disorders? Testing validity issues in a clinical sample. Int J Methods Psychiatr Res 1998;7:142-55.

[18] Cohen S, Tyrrell DA, Russell MA, Jarvis MJ, Smith AP. Smoking, alcohol consumption, and susceptibility to the common cold. Am J Public Health 1993;83:1277-83.

[19] Winkler J, Stolzenberg H. Der Sozialschitenindex im BundesGesundheitssurvey. Gesundheitswesen 1998;60:21-6.

[20] Coxe S, West SG, Aiken LS. The analysis of count data: a gentle introduction to poisson regression and its alternatives. J Pers Assess 2009;91:121-36.

[21] Hilbe JM. Negative binomial regression. 2nd ed. Cambridge: Cambridge university press; 2011.

[22] Stata Library: Categorical and count data analysis utilities. http://www.ats.ucla.edu/stat/stata/library/longutil.htm.

(Accessed September 7, 2011). Los Angeles: UCLA: Academic Technology Services.

[23] Stata Corporation. Stata statistical software: release 10. TX: Stata Corporation; 2007.

[24] Chaloner K, Brant R. A Bayesian approach to outlier detection and residual analysis. Biometrika 1988;75:651-59.

[25] Kiecolt-Glaser JK, Glaser R. Depression and immune function: central pathways to morbidity and mortality. J Psychosom Res 2002;53:873-6. 
[26] Cohen S, Herbert TB. Health psychology: psychological factors and physical disease from the perspective of human psychoneuroimmunology. Annu Rev Psychol 1996;47:113-42.

[27] Aldwin CM. Stress, coping, and development: an integrative perspective. New York: The Guilford Press; 1994.

[28] Sareen J, Jacobi F, Cox BJ, Belik SL, Clara I, Stein MB. Disability and poor quality of life associated with comorbid anxiety disorders and physical conditions. Arch Intern Med 2006;166:2109-16.

[29] Glaser R, Kiecolt-Glaser JK. Stress-induced immune dysfunction: implications for health. Nat Rev Immunol 2005;5:243-51.

[30] Salovey P, Rothman AJ, Detweiler JB, Steward WT. Emotional states and physical health. Am Psychol 2000;55:110-21.

[31] Kiecolt-Glaser JK, Christian L, Preston H, Houts CR, Malarkey WB, Emery $\mathrm{CF}$, et al. Stress, inflammation, and yoga practice. Psychosom Med 2010;72:113-21.

[32] Salovey P, Birnbaum D. Influence of mood on health-relevant cognitions. J Pers Soc Psychol 1989;57:539-51.

[33] Houtveen JH, van Doornen LJ. Medically unexplained symptoms and between-group differences in 24-h ambulatory recording of stress physiology. Biol Psychol 2007;76:239-49.

[34] Cohen S, Doyle WJ, Turner RB, Alper CM, Skoner DP. Emotional style and susceptibility to the common cold. Psychosom Med 2003;65:652-7.

[35] Macintyre S, Pritchard C. Comparisons between the self-assessed and observer-assessed presence and severity of colds. Soc Sci Med 1989;29:1243-8. 
[36] Dantzer R, O'Connor JC, Freund GG, Johnson RW, Kelley KW. From inflammation to sickness and depression: when the immune system subjugates the brain. Nat Rev Neurosci 2008;9:46-56.

[37] Kunik ME, Roundy K, Veazey C, Souchek J, Richardson P, Wray NP, et al. Surprisingly high prevalence of anxiety and depression in chronic breathing disorders. Chest 2005;127:1205-11.

[38] Dahlen I, Janson C. Anxiety and depression are related to the outcome of emergency treatment in patients with obstructive pulmonary disease. Chest 2002;122:1633-7.

[39] Moussas G, Tselebis A, Karkanias A, Stamouli D, llias I, Bratis D, et al. A comparative study of anxiety and depression in patients with bronchial asthma, chronic obstructive pulmonary disease and tuberculosis in a general hospital of chest diseases. Annals of general psychiatry 2008;7:7.

[40] Mikkelsen RL, Middelboe T, Pisinger C, Stage KB. Anxiety and depression in patients with chronic obstructive pulmonary disease (COPD). A review. Nordic journal of psychiatry 2004;58:65-70.

[41] Yohannes AM, Baldwin RC, Connolly MJ. Depression and anxiety in elderly patients with chronic obstructive pulmonary disease. Age Ageing 2006;35:457-9.

[42] Fink P, Orbol E, Hansen MS, Sondergaard L, De Jonge P. Detecting mental disorders in general hospitals by the SCL-8 scale. J Psychosom Res 2004;56:371-5.

[43] Vila M, Kramer T, Obiols JE, Garralda ME. Adolescents who are frequent attenders to primary care: contribution of psychosocial factors. Soc Psychiatry Psychiatr Epidemiol 2012;47:323-9. 
[44] Lobo E, De Jonge P, Huyse FJ, Slaets JP, Rabanaque MJ, Lobo A. Early detection of pneumology inpatients at risk of extended hospital stay and need for psychosocial treatment. Psychosom Med 2007;69:99-105.

[45] Gudmundsson G, Gislason T, Janson C, Lindberg E, Hallin R, Ulrik CS, et al. Risk factors for rehospitalisation in COPD: role of health status, anxiety and depression. Eur Respir J 2005;26:414-9.

[46] Garuti G, Cilione C, Dell'Orso D, Gorini P, Lorenzi MC, Totaro L, et al. Impact of comprehensive pulmonary rehabilitation on anxiety and depression in hospitalized COPD patients. Monaldi Arch Chest Dis 2003;59:56-61. 


\section{Table 1}

Sociodemographic characteristics of the study sample $(N=4022)$

$n$

Gender

Male

Female

2001

49.7

Age

Mean (41.8), SD (13.1)

Marital status

In relationship

2583

64.2

Not in relationship

1439

35.8

Socioeconomic status

Middle/high

3265

81.2

Low
18.8

$S D$ standard deviation 


\section{Table 2}

Crude and adjusted incidence rate ratios (IRRs) of cold episodes for DSM-IV mental disorders compared to the reference group that had no indexed mental disorder during the past 12 months $(N=$ 4022)

\begin{tabular}{|c|c|c|c|c|}
\hline$D S M-I V$ mental disorder $(n)$ & IRR c & $95 \% \mathrm{Cl}$ & IRR a & $95 \% \mathrm{Cl}$ \\
\hline Any $D S M-I V$ mental disorder (1555) & $1.52^{*}$ & $(1.37-1.70)$ & $1.44^{*}$ & $(1.29-1.60)$ \\
\hline Any substance abuse or dependence (547) & $1.44^{*}$ & $(1.24-1.66)$ & $1.32^{*}$ & $(1.14-1.52)$ \\
\hline Alcohol abuse or dependence (169) & 1.17 & $(0.92-1.50)$ & 1.13 & $(0.88-1.45)$ \\
\hline Nicotine dependence (425) & $1.41^{*}$ & $(1.20-1.66)$ & $1.28^{*}$ & $(1.10-1.50)$ \\
\hline Illicit substance abuse or dependence (29) & $3.16^{*}$ & $(2.27-4.40)$ & $2.39^{*}$ & $(1.73-3.30)$ \\
\hline Possible psychotic (114) & $1.53^{*}$ & $(1.15-2.03)$ & $1.43^{*}$ & $(1.09-1.87)$ \\
\hline Any mood (536) & $1.42^{*}$ & $(1.22-1.65)$ & $1.35^{*}$ & $(1.16-1.56)$ \\
\hline Major depression (370) & $1.47^{\star}$ & $(1.24-1.74)$ & $1.39^{*}$ & $(1.17-1.64)$ \\
\hline Dysthymia (212) & $1.32^{*}$ & $(1.06-1.64)$ & $1.34^{*}$ & $(1.07-1.68)$ \\
\hline Bipolar (39) & 1.47 & $(0.85-2.53)$ & 1.21 & $(0.72-2.01)$ \\
\hline Any anxiety (695) & $1.48^{*}$ & $(1.30-1.69)$ & $1.40^{*}$ & $(1.23-1.59)$ \\
\hline Panic (113) & $1.67^{*}$ & $(1.24-2.24)$ & $1.57^{\star}$ & $(1.19-2.08)$ \\
\hline Agoraphobia (97) & $1.44^{*}$ & $(1.05-1.98)$ & $1.47^{\star}$ & $(1.06-2.04)$ \\
\hline Social phobia (89) & $1.48^{*}$ & $(1.08-2.01)$ & 1.32 & $(0.97-1.79)$ \\
\hline Specific phobia (369) & $1.63^{*}$ & $(1.39-1.92)$ & $1.52^{*}$ & $(1.29-1.78)$ \\
\hline Generalized anxiety (67) & 1.27 & $(0.82-1.95)$ & 1.28 & $(0.86-1.91)$ \\
\hline Obsessive-compulsive (33) & 1.43 & $(0.87-2.35)$ & 1.32 & $(0.80-2.15)$ \\
\hline Any somatoform (467) & $1.42^{*}$ & $(1.21-1.67)$ & $1.38^{*}$ & $(1.18-1.62)$ \\
\hline Somatic symptom index 4/6 (182) & $1.32^{*}$ & $(1.03-1.69)$ & $1.39^{*}$ & $(1.09-1.76)$ \\
\hline Pain (339) & $1.31^{*}$ & $(1.08-1.58)$ & $1.26^{\star}$ & $(1.05-1.51)$ \\
\hline
\end{tabular}

Cl confidence interval; DSM-IV Diagnostic and Statistical Manual of Mental Disorders, Fourth Edition; IRR a adjusted incidence rate ratios for age, gender, and marital, and socioeconomic status; IRR $c$ crude incidence rate ratios ${ }^{*} p<.05$ 